Mélanges

de l'École française de Rome

Moyen Âge

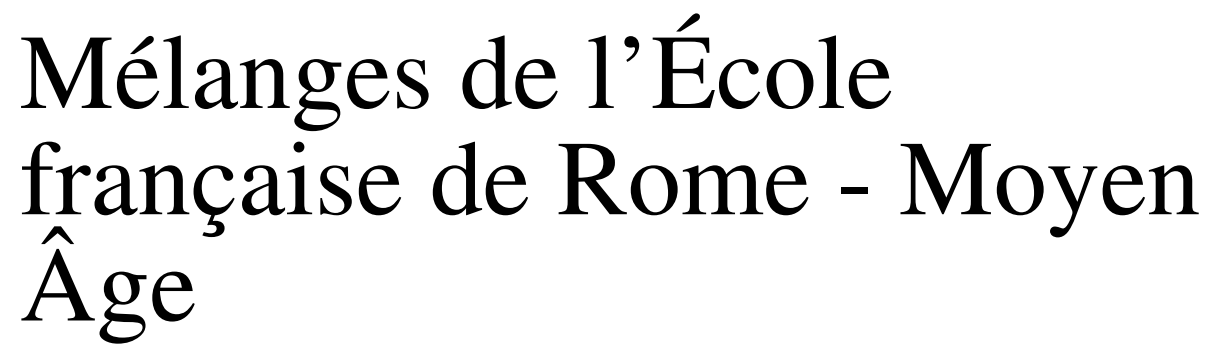

127-2 (2015)

Italians and Eastern Europe in Late Middle Ages / Les Italiens et l'Europe orientale au Bas Moyen Âge / Italiani ed Europa orientale nel basso Medioevo - Varia - Ateliers doctoraux - Regards croisés

Cristina Carbonetti, Arianna Cervi, Marta De Bianchi et Jean-Marie Martin

\title{
Les cartulaires ecclésiastiques de l'Italie médiévale
}

\begin{abstract}
Avertissement
Le contenu de ce site relève de la législation française sur la propriété intellectuelle et est la propriété exclusive de l'éditeur.

Les œuvres figurant sur ce site peuvent être consultées et reproduites sur un support papier ou numérique sous réserve qu'elles soient strictement réservées à un usage soit personnel, soit scientifique ou pédagogique excluant toute exploitation commerciale. La reproduction devra obligatoirement mentionner l'éditeur, le nom de la revue, l'auteur et la référence du document.

Toute autre reproduction est interdite sauf accord préalable de l'éditeur, en dehors des cas prévus par la législation en vigueur en France.
\end{abstract}

\section{revues.org}

Revues.org est un portail de revues en sciences humaines et sociales développé par le Cléo, Centre pour l'édition électronique ouverte (CNRS, EHESS, UP, UAPV).

Référence électronique

Cristina Carbonetti, Arianna Cervi, Marta De Bianchi et Jean-Marie Martin, «Les cartulaires ecclésiastiques de

I'Italie médiévale », Mélanges de l'École française de Rome - Moyen Âge [En ligne], 127-2 | 2015, mis en ligne le 16 octobre 2015, consulté le 29 octobre 2015. URL : http://mefrm.revues.org/2655

Éditeur : École française de Rome

http://mefrm.revues.org

http://www.revues.org

Document accessible en ligne sur :

http://mefrm.revues.org/2655

Document généré automatiquement le 29 octobre 2015

(c) École française de Rome 


\section{Cristina Carbonetti, Arianna Cervi, Marta De Bianchi et Jean-Marie Martin}

\section{Les cartulaires ecclésiastiques de l'Italie médiévale}

«Un cartulaire (lat. : $c(h)$ artularium) est un recueil de copies de ses propres documents, établi par une personne physique ou morale, qui, dans un volume ou plus rarement dans un rouleau, transcrit ou fait transcrire intégralement ou parfois en extraits des titres relatifs à ses biens et à ses droits et des documents concernant son histoire ou son administration, pour en assurer la conservation et en faciliter la consultation. Un tel recueil, étant établi par l'intéressé luimême, ne présente pas nécessairement une présomption de sincérité pour les actes qui y sont transcrits, et pas davantage de valeur authentique, à moins que, après collation, des marques extérieures d'authenticité n'y soient portées par une autorité habilitée à les leur conférer » ${ }^{1}$. Cette définition, globalement satisfaisante, appelle toutefois quelques remarques. En premier lieu, le but assigné au cartulaire par le Vocabulaire (assurer la conservation et faciliter la consultation des documents) n'est pas toujours assuré ; il faut aussi compter avec la simple mémoire de l'établissement et son désir de la faire connaître et de l'exalter : nous renvoyons à ce sujet, par exemple, au récent ouvrage consacré à la présentation et à la décoration du Chronicon Casauriense ${ }^{2}$. Comme l'écrit Patrick Geary à propos des cartulaires ecclésiastiques de la Francie orientale des IX $^{\mathrm{e}}-\mathrm{XII}{ }^{\mathrm{e}}$ siècles ${ }^{3}$, ces recueils n'ont pas eu pour seul but pratique de conserver les documents de l'institution ; ils ont aussi servi à en défendre les droits et à transmettre la mémoire des bienfaiteurs, des abbés et des évêques, ce qui a influé sur leurs caractères externes et internes, orientant les principes d'exclusion et d'inclusion des documents et favorisant des pratiques de falsification.

De tout cela résulte que les cartulaires constituent pour le médiéviste, surtout en Occident, une catégorie de documents de toute première importance. Chaque cartulaire présente un double intérêt. D'une part il transmet des actes dont l'original est le plus souvent perdu, notamment des actes du très haut Moyen Âge (par exemple, le Chronicon Sanctae Sophiae fait connaître plus de 130 actes du VIII ${ }^{\mathrm{e}}$ siècle, ce qui est considérable ${ }^{4}$ ). En outre, la décision même de faire une copie (ou de résumer) des documents dans un certain ordre (ou parfois sans ordre bien clair) et la façon dont elle est mise en pratique nous renseignent sur la conservation et la gestion des biens et du patrimoine archivistique et sur les crises qui surviennent. Un tel travail présente un aspect idéologique, traduisant la mémoire de l'établissement qui l'entreprend et sa conscience historique $^{5}$ : la compilation d'un cartulaire résulte toujours d'un choix, qui reflète parfois très clairement les motivations qui ont poussé à sa compilation ; en revanche, l'utilité pratique est extrêmement variable. Le résultat peut donc être apprécié à la fois par rapport à la motivation et du point de vue de son efficacité.

\section{État de la question}

Dans les pays de l'Europe du Nord, qui n'ont créé que très tard un notariat public, l'importance relative des cartulaires dans la documentation, surtout en ce qui concerne le Moyen Âge central, est considérable. En effet, la légitimité de la copie de documents qui se présentent souvent sous une forme diplomatique assez fruste ne pose pas de problème; par conséquent, la conservation d'actes uniquement dans des cartulaires n'en altère pas vraiment la valeur. C'est pourquoi, dans ces pays, les recherches sur les cartulaires, les répertoires et les éditions ont été entrepris depuis longtemps.

Ainsi, en France, il y a plus d'un siècle, Henri Stein a publié une bibliographie générale des cartulaires français qui fait toujours autorité ${ }^{6}$. Il s'agit d'un simple répertoire, visant à rassembler tous les cartulaires. Après la publication de ce volume, il a préparé un supplément recensant environ 1200 nouveaux numéros, qui n'a pas été publié 7 . Le travail a été repris, dans les années 1960, par Jacqueline Le Braz, qui s'est intéressée aux diocèses de Laon, Soissons 
et Châlons ${ }^{8}$, mais la publication est restée assez confidentielle. En 1965, une commission avait prévu la refonte du Stein, en deux volumes, l'un consacré aux cartulaires ecclésiastiques, un autre aux laïques. Plus récemment, l'Institut de Recherche et d'Histoire des Textes a entrepris d'ajourner et surtout d'approfondir le travail de Stein. Un premier volume, couvrant le SudEst de la France, a été publiế ; il ne se contente pas d'énumérer les cartulaires, mais, grâce à une fiche signalétique, permet d'en établir une typologie. Des répertoires de cartulaires ont été publiés depuis longtemps en Belgique ${ }^{10}$ et, plus récemment, en Angleterre ${ }^{11}$. La situation est moins claire en Allemagne, où il n'existe pas de travail d'ensemble ${ }^{12}$. Enfin, il n'existe rien sur l'Italie ni sur la péninsule ibérique (où une équipe travaille en ce moment sur le sujet), pays de notariat. On avait bien tenté, à la fin du $\mathrm{XIX}^{\mathrm{e}}$ siècle, une présentation générale des cartulaires européens ${ }^{13}$, et donc notamment italiens ${ }^{14}$. Mais Henri Stein, qui avait évidemment consulté cet ouvrage, écrit ${ }^{15}$ : « Je mentionne à peine le Wegweiser durch die Literatur der UrkundenSammlungen d'Hermann Oesterley, qui a prétendu indiquer tous les cartulaires existants, manuscrits et imprimés ; l'auteur, insuffisamment préparé à ce travail pour lequel il s'est dispensé de toute recherche personnelle, s'est malheureusement fourvoyé trop souvent pour pouvoir être utilement consulté ». De fait, si Oesterley a vu beaucoup de choses, sa typologie est très défectueuse.

L'exemple français (le plus important à ce jour) montre d'abord combien il est difficile de recenser l'ensemble des cartulaires à l'échelle d'un pays : même l'ouvrage de Stein, prototype de telles recherches, est incomplet et son auteur s'en était rendu compte ; nous avons bien conscience que, dans un tel catalogue, les lacunes sont inévitables; encore convient-il qu'elles ne soient ni trop nombreuses, ni trop importantes.

La bibliographie récente déjà signalée, en France et aussi en Angleterre, montre que l'édition et l'étude des cartulaires, inaugurées à l'époque moderne, reprises au début du XX $\mathrm{X}^{\mathrm{e}}$ siècle, ont connu depuis quelques décennies un regain d'intérêt. On doit citer à ce propos un ouvrage important : les actes du colloque tenu en 1991 à Paris, à l'École des Chartes, publiés en 1993 par Olivier Guyotjeannin, Laurent Morelle et Michel Parisse ${ }^{16}$. Ce regain d'intérêt a eu pour effets non seulement d'augmenter le nombre des cartulaires répertoriés, et même édités, mais aussi de déplacer le regard porté sur ces sources. Le cartulaire est passé du statut de simple réservoir de documents (ce qu'il est aussi) à celui d'œuvre autonome, objet d'étude pour lui-même.

\section{Le cas italien}

Il n'existe donc pas, à ce jour, pour l'Italie, de répertoire comparable à celui d'Henri Stein pour la France. De plus, l'exemple italien impose quelques nuances à la définition même de l'objet de notre programme de recherche.

À vrai dire, quand on évoque les cartulaires italiens, on envisage généralement deux catégories de sources assez différentes l'une de l'autre - auxquelles on peut en ajouter une troisième, comme on va le voir.

La première est constituée par la série des cartulaires monastiques compilés à partir de la seconde moitié du $\mathrm{XI}^{\mathrm{e}}$ siècle, à l'époque de la réforme de l'Église romaine. Ceux qui ont été établis dans les scriptoria monastiques, en particulier, ressemblent beaucoup à ceux de l'Europe septentrionale. Certains s'en distinguent toutefois d'une part par leur ampleur, d'autre part parce qu'ils sont liés à des œuvres historiques et/ou canoniques qui souvent se trouvent dans le même volume. Ces « cartulaires-chroniques » ou ensembles de cartulaires et d'œuvres historiques faits au même moment dans le même établissement sont bien connus ${ }^{17}$. À Farfa, le moine Grégoire de Catino a d'abord compilé, à partir de 1092, le cartulaire des possessions de l'abbaye (Regestum Farfense, officiellement intitulé Liber gemniographus sive

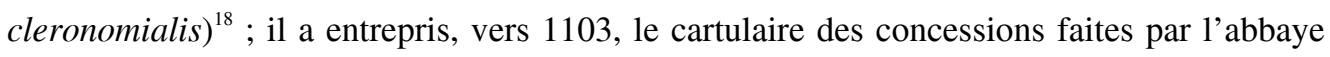

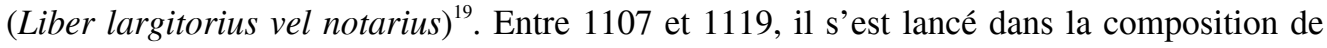
la Chronique ${ }^{20}$. En 1130, enfin, il donnait le Liber Floriger ${ }^{21}$, index alphabétique général de son œuvre. La démarche suivie au même moment au Mont-Cassin est formellement inverse : 
vers 1099, Léon Marsicanus, bibliothécaire et archiviste du Mont-Cassin, devenu peu après (entre 1102 et 1107) cardinal évêque d'Ostie, entame à la demande de l'abbé Oderisius I ${ }^{\text {er }}$ les Chronica monasterii Casinensis, qu'il laisse inachevés à sa mort, le 22 mai 1115, mais qui sont ensuite complétés par le moine Gui, puis par Pierre Diacre ${ }^{22}$, qui avait déjà, entre 1131 et 1133, entrepris de diriger la compilation d'un cartulaire monumental, appelé Registrum Petri Diaconi $^{23}$. Le Liber preceptorum de S. Sofia de Bénévent (ou Chronicon Sanctae Sophiae) a été terminé en 1119 par un moine anonyme (peut-être Iohannes Grammaticus, élu abbé en $1120)^{24}$. Le volume comprend, dans l'ordre : des annales (de la naissance du Christ à 1119 , ensuite prolongées jusqu'en 1128), une collection canonique, un catalogue des ducs et princes de Bénévent, enfin un cartulaire nettement divisé en six sections, qui ne contient que des actes publics. Le Chronicon Vulturnense, fait sous la direction d'un moine Jean dont on ne sait rien $^{25}$, a été entrepris entre 1119 et 1124 . Dans ce cas c'est la chronique - au plan complexe et intégrant des éléments remployés — qui sert de fil conducteur ; les documents sont fournis dans son cadre, entrecoupés de textes de liaison. Le Chronicon Casauriense $e^{26}$ a été composé par Iohannes Berardi et écrit par le magister Rusticus ; il été fait entre 1179 et 1182 et couvre la période qui va de la fondation de l'abbaye (871) à la mort de l'abbé Leonas (1182). Cette œuvre est certainement la plus originale par sa présentation synoptique des documents et de la chronique. Dernier grand cartulaire-chronique : celui de S. Bartolomeo di Carpineto ${ }^{27}$, terminé entre septembre 1194 et avril 1195 (et augmenté peu après). Il comprend, après le prologue et une table des capitula, une chronique en six livres couvrant la période 962-1194, des documents, enfin une dédicace en vers.

L'autre catégorie est celle des libri iurium ou cartulaires communaux, qui sont des recueils documentaires voulus et organisés sous forme de livres à l'initiative des organes communaux, dans le but d'y verser tous les documents qui attestaient les droits de la cité, les iura communis, les prérogatives politiques, juridictionnelles et patrimoniales de l'institution communale, en d'autres termes les fondements juridiques de son existence. Leur origine était justifiée par des considérations à la fois idéologiques et pratiques : préserver la mémoire historique, politique et juridictionnelle de la Commune en créant un monument à la gloire de la cité, et en même temps réorganiser et sauvegarder les documents les plus anciens et rationaliser la conservation des plus récents. L'étude de cette documentation a connu un moment privilégié au début du $\mathrm{XX}^{\mathrm{e}}$ siècle, où de nombreux cartulaires communaux ont été publiés, notamment dans le cadre régional des «Società/Deputazioni di storia patria ». Ensuite, au cours des trente dernières années, les travaux de Dino Puncuh et d'Antonella Rovere ${ }^{28}$ ont renouvelé l'attention pour ce type d'écrits, inaugurant une nouvslle série d'études et suscitant de nouvelles recherches centrées sur des communes particulières, ou visant à analyser le phénomène des libri iurium dans des cadres plus larges, régional ou supra-régional, ou encore à le situer dans des perspectives plus vastes, comme, par exemple, le rapport entre écriture documentaire et activité gouvernementale. Toutes ces recherches ont permis, au cours des trois dernières décennies, d'ajouter beaucoup de nouvelles pièces à une marqueterie qui s'est progressivement composée et développée, contribuant à préciser toujours plus les aspects principaux d'un phénomène documentaire de grandes dimensions qui a traversé toute la civilisation communale, depuis le nord de la péninsule jusqu' aux confins méridionaux de l'État pontifical.

Enfin, si nous considérons bien les « condaghi » sardes comme des cartulaires, nous en faisons une catégorie particulière, bien distincte, que nous traiterons à part.

\section{Perspectives de recherche}

Notre décision d'entreprendre une recherche sur les cartulaires ecclésiastiques de l'Italie médiévale est née d'une double constatation et d'une exigence méthodologique : en premier lieu le hiatus considérable qui sépare ce type de cartulaires des cartulaires communaux, et qui empêche d'étudier le phénomène des cartulaires italiens en bloc ; en second lieu le fait que, alors que sur les cartulaires communaux les recherches sont déjà importantes, les cartulaires ecclésiastiques semblent, pour le moment, susciter peu d'études ; ils sont mal connus, tant en ce qui concerne leur importance qu'en tant que phénomène documentaire à considérer 
comme tel. Enfin, limiter notre recherche aux cartulaires d'établissements ecclésiastiques et, plus précisément, à ceux qui ont été compilés au Moyen Âge (c'est à dire avant la fin du $\mathrm{XV}^{\mathrm{e}}$ siècle) signifie que nous les étudions non de façon abstraite, comme conteneurs plus ou moins volumineux de documents, mais bien comme les produits de l'activité documentaire du Moyen Âge, et par conséquent en tant que phénomène historique qui doit être replacé dans le milieu qui les a vu naître, cherchant à mettre en lumière les buts qui, cas par cas, ont orienté leur réalisation.

Nous prenons en compte, outre ceux qui sont conservés, ceux qui ne le sont qu'en partie, ou encore sous forme de copies (copies de cartulaires, pas d'actes isolés), enfin ceux qui sont complètement perdus, mais dont l'existence est attestée.

À ce projet, initié par Cristina Carbonetti et Jean-Marie Martin, ont été associées, dès l'abord, deux jeunes chercheuses qui s'intéressaient déjà au sujet, sous la conduite de Cristina Carbonetti : Arianna Cervi et Marta De Bianchi qui vient de publier un excellent répertoire commenté des cartulaires de la région du Latium (entrepris avant le début de notre programme $)^{29}$; cet ouvrage constitue la première base régionale solide pour nos recherches. Ont en outre participé — ou doivent participer — à nos travaux plusieurs personnes, qui nous ont donné leur accord.

Mais nous devons surtout rechercher des correspondants locaux dans les différentes régions italiennes $^{30}$, qui puissent d'une part nous renseigner sur des cartulaires inconnus ou mal définis, et d'autre part appliquer notre grille à des ouvrages inédits ; on a notamment pensé à ceux qui se sont déjà livrés à des études régionales sur le sujet ${ }^{31}$. On demandera aux correspondants régionaux non seulement des recherches ponctuelles, mais aussi une approche synthétique des cartulaires de leur région, afin de pouvoir réaliser le seconde phase de la recherche.

Le premier stade de cette recherche prévoit le recensement global des cartulaires ecclésiastiques, afin de préparer un répertoire aussi complet que possible, qui affecte à chacun une place typologique, grâce à une fiche descriptive.

Évidemment, l'établissement de la fiche signalétique de chaque cartulaire - qu'on s'efforcera de rendre utilisable également par l'informatique — vise à une précision bien supérieure à la définition des grandes catégories qu'on présente ci-dessous. On s'attachera à la description codicologique du cartulaire, au nombre de documents transcrits, à leur typologie, à la date de compilation du cartulaire et aux dates limites des actes qui y sont copiés ou résumés ; on relèvera les causes officielles de la compilation, quand elles sont indiquées ; on étudiera le mode de classement des actes, l'éventuelle division du cartulaire en sections et la valeur pratique de sa présentation ; on évaluera, dans la mesure du possible, le mode de transcription et la fidélité de la copie (qu'on a pu récemment apprécier dans le cas du Registrum Petri Diaconi, dont le quart des documents est conservé en originaux). On s'attachera à la décoration, quand elle existe ${ }^{32}$. On indiquera les éventuelles parties du volume qui n'appartiennent pas au cartulaire. On signalera, évidemment, les éventuelles copies du cartulaire, les éditions et la bibliographie.

Mais la première tâche, qui n'est ni la plus aisée, ni la plus stimulante, consiste à repérer, dans les archives et bibliothèques italiennes, le plus grand nombre possible de cartulaires : un certain nombre sont à peu près ignorés, voire inconnus. On a commencé par dépouiller de grands répertoires (Kehr, Mazzatinti ${ }^{33}$ ) ; on doit encore dépouiller systématiquement les grandes revues régionales (notamment du type Archivio storico).

À l'issue de cette première phase, il sera possible, grâce au répertoire qui aura été réalisé, d'étudier le phénomène dans sa complexité et dans sa globalité.

Notre projet a été admis au nombre des programmes de l'École française de Rome, où nous nous réunissons tous les ans (29 décembre 2011, 10 décembre 2012, 13 juin 2013, 22 mai et 24 novembre 2014 ; deux réunions sont déjà prévues en 2015). Nous devons bénéficier d'un financement du Labex REXMED.

\section{Résultats escomptés}

Quand on tente d'envisager le problème dans sa globalité, on s'aperçoit rapidement que l'Italie présente, au Moyen Âge, des conditions spécifiques qui influent sur la confection des 
cartulaires. Certes, comme partout, les grands établissements religieux (et quelques plus petits) sont par moments tentés de copier leurs archives dans des ensembles plus ou moins cohérents ; comme partout, la réforme de l'Église romaine à la fin du $\mathrm{XI}^{\mathrm{e}}$ siècle les a poussés à accorder une attention particulière à leur temporel, canoniquement inaliénable ; les crises qui les frappent peuvent avoir le même effet. On a toutefois montré, à propos des grands cartulaires de l'Italie centro-méridionale du $\mathrm{XII}^{\mathrm{e}}$ siècle, que la compilation de chacun avait des causes contingentes plus précises. En outre, globalement, l'Italie présente deux particularités notables. La première est la présence de quelques églises disposant d'un temporel véritablement énorme, dont les bases remontent à l'Antiquité chrétienne, comme l'Église de Rome et celle de Ravenne. La seconde - qui nous paraît plus importante - est la présence, dans toute l'Italie et pendant tout le Moyen Âge, d'un notariat public, dont les compétences se précisent au fil du temps, mais qui, dès le haut Moyen Âge, a le monopole de la documentation privée. D'où le fait que, en Italie, la situation des cartulaires est plus variée, puisqu'ils transmettent des actes aussi bien en copie simple qu'en copie établie par un notaire. En outre, au bas Moyen Âge (à partir du $\mathrm{XII}^{\mathrm{e}}$ siècle), dans l'Italie centro-septentrionale, les communes font elles-mêmes des sortes de cartulaires (les libri iurium), compilés par des notaires : cette pratique influe sur la confection des cartulaires ecclésiastiques ; on distingue donc déjà deux «générations » de cartulaires assez bien différenciées. La première génération, de loin la mieux étudiée, est constituée par les cartulaires de l'époque de la réforme, compilés du $\mathrm{XI}^{\mathrm{e}}$ au XIII ${ }^{\mathrm{e}}$ siècle et principalement au XII ${ }^{\mathrm{e}}$. Établis, comme les précédents, à l'intérieur de l'établissement religieux (le plus souvent, donc, par des moines) dont le cartulaire vise à rassembler les biens et les droits, ils sont très divers dans le détail. Ils contiennent parfois seulement des actes publics (libri preceptorum, comme le Chronicon Sanctae Sophiae), parfois aussi des actes privés enregistrant des acquisitions auprès de particuliers - principalement des offrandes (comme, par exemple, le Registrum Petri Diaconi). Appartiennent notamment à cette grande catégorie les cartulaires-chroniques, déjà évoqués. Même si la plupart ont été compilés dans de très grandes abbayes, certains établissements plus modestes en ont produit (S. Bartolomeo de Carpineto, mais aussi S. Salvatore a Maiella); ils servent de support unique à la mémoire de l'établissement. On voit que cette catégorie est complexe.

Seconde génération : à partir du XIII ${ }^{\mathrm{e}}$ siècle apparaissent, du moins dans l'Italie communale ${ }^{34}$, les cartulaires compilés par des notaires et mêlant parfois dans le même volume actes originaux insérés, copies figuratives, copies authentiques et copies simples (mais seulement en apparence, puisqu'en réalité elles bénéficient d'une forme d'authentication générale attribuée à l'ensemble du volume), à la façon des libri iurium que les communes développent à la même époque. Deux importantes études se sont déjà attachées à ce genre de cartulaires dans le cadre ligure $^{35}$.

En outre, on peut peut-être indivdualiser dès à présent (mais la réponse définitve ne sera fournie qu'au terme de la recherche) les caractéristiques d'une génération antérieure aux deux autres.

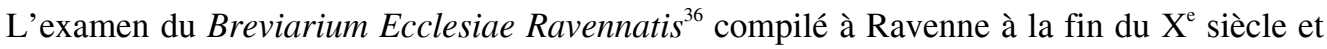
d'autres petites listes d'actes éditées avec lui, ainsi que la récente découverte du Breve de multis pensionibus de Lucques ${ }^{37}$ nous ont semblé démontrer que la plus ancienne forme connue de cartulaires italiens (qui apparaît, en tout cas, à la fin du IX ${ }^{\mathrm{e}}$ siècle) était la compilation non d'actes d'offrandes ou d'autres acquisitions, mais de contrats de concession (emphytéoses, «livelli »).

Les cartulaires italiens sont de toute taille et de toute importance : le Chronicon Casauriense contient plus de 2000 documents, le Registrum Petri Diaconi plus de 700, alors que certaines listes d'actes ravennates en ont moins de dix. La plupart se présentent sous la forme de codices de parchemin, mais tel n'est pas le cas général. Passons rapidement sur les copies sur papier. Certains cartulaires de haute époque sont écrits sur papyrus, matériau qui a été utilisé jusqu'au XI ${ }^{\text {e }}$ siècle dans certaines zones de tradition byzantine (dont Rome) ${ }^{38}$ : l'exemple le plus connu est le Breviarium Ecclesiae Ravennatis, déjà cité. D'autres cartulaires se présentent 
sous la forme de rouleaux de parchemin, de Novare dans le Piémont ${ }^{39}$ à Oppido en Calabre ${ }^{40}$. D'autre part, certains cartulaires contiennent surtout des actes d'acquisitions patrimoniales (donations, testaments, achats, concessions publiques etc.) ; d'autres en revanche, comme le Breviarium Ecclesiae Ravennatis et le Breve de multis pensionibus rassemblent une documentation qui concerne la gestion des patrimoines. D'autres différences paraissent dues à la tradition des documents (originaux, ou copies simples, authentiques, imitatives) et aussi au type d'institution religieuse qui les a fait établir (monastères, chapitres, évêchés).

L'objectif de notre recherche est précisément de mettre en évidence ces différences grâce au recensement général des cartulaires ecclésiastiques : seule en effet la connaissance de leur répartition chronologique et géographique et de leur diversité typologique en ce qui concerne le contenu, la tradition des actes, les rédacteurs et les institutions qui les ont produits, pourra permettre de reconstituer l'évolution de cet important phénomène documentaire et historique et, finalement, d'approfondir les relations des églises avec leur temporel, avec leur histoire et avec les institutions publiques. On espère aussi qu'une telle recherche fera émerger quelques sources nouvelles.

\section{Bibliographie}

Allegria 2006 : S. Allegria, Un rotolo-cartulario dell'Archivio capitolare di Arezzo. Tra documentazione e storia, dans S. Allegria, F. Cenni (éd.), Secoli XI e XII: l'invenzione della memoria. Atti del Seminario Internazionale, Montepulciano, 27-29 aprile 2006, Montepulciano, 2006, p. 103-112.

Balzani (éd.) 1903 : Il Chronicon Farfense di Gregorio di Catino. Precedono la Constructio Farfensis $e$ gli scritti di Ugo di Farfa, éd. U. Balzani, Rome, 1903, 2 vol. (Fonti per la storia d'Italia, 33-34).

Cameli 2002 : M. Cameli, Sulle tracce del « liber catasti » della Chiesa ascolana : una ipotesi di ricostruzione, dans Rivista di storia della Chiesa in Italia, 56, 2002, p. 349-376.

Cameli 2003 : M. Cameli, Studi preliminari sui registri vescovili di Ascoli Piceno, dans A. Bartoli Langeli, A. Rigon (éd.) I registri vescovili dell'Italia settentrionale (secoli XIII-XV). Atti del Convegno di studi (Monselice, 24-25 novembre 2000), Rome, 2003, p. 373-401.

Cameli 2005 : M. Cameli, Note di diplomatica vescovile duecentesca. Frammenti di registri vescovili nell'Archivio capitolare di Ascoli Piceno, dans Rassegna degli archivi di Stato, n. s. 1-2, 2005, p. 170-201.

Cameli 2009 : M. Cameli, La chiesa scritta. Documentazione e autorappresentazione dei vescovi di Ascoli Piceno tra XI e XIII secolo, Verona, 2009 (Biblioteca dei Quaderni di storia religiosa, 6).

Carbonetti Vendittelli 2011 : C. Carbonetti Vendittelli, I supporti scrittorii della documentazione : l'uso del papiro, dans J.-M. Martin, A. Peters Custot, V. Prigent (éd.), L'héritage byzantin en Italie (VIII XII siècle). I. La fabrique documentaire, Rome, 2011 (Collection de l'École française de Rome, 449), p. 33-48.

Cárcel Orti (éd.) 1997 : Commission internationale de diplomatique. Comité international des sciences historiques. Vocabulaire international de la diplomatique, M. Milagros Cárcel Ortí (éd.), Valence, 1997.

Davis 1958 : G. R. C. Davis, Medieval Cartularies of Great Britain : a Short Catalogue, Londres, 1958.

De Bianchi 2013 : M. De Bianchi, I cartulari medievali del Lazio, Tivoli, 2013.

Federici (éd.) 1925-1940 : Il Chronicon Vulturnense del monaco Giovanni, éd. V. Federici, Rome, 1925-1940, 3 vol. (Fonti per la storia d'Italia, 58-60).

Feller 1993 : L. Feller, Le cartulaire-chronique de S. Clemente a Casauria, dans Guyotjeannin, Morelle, Parisse 1993, p. 261-272.

Fuselli (éd.) 1996 : Il Chronicon di S. Bartolomeo di Carpineto, éd. E. Fuselli, L’Aquila, 1996.

Geary 1996 : P. T. Geary, La mémoire et l'oubli à la fin du premier millénaire, Paris, 1996.

Giorgi, Balzani (éd.) 1879-1914 : Il Regesto di Farfa, compilato da Gregorio di Catino e pubblicato dalla Società Romana di Storia Patria, éd. I. Giorgi et U. Balzani, Rome, 1879-1914, 5 vol.

Guillou (éd.) 1972 : A. Guillou, La Théotokos de Hagia-Agathè (Oppido) (1050-1064/1065), Cité du Vatican, 1972 (Corpus des actes grecs d'Italie du Sud et de Sicile, 3).

Guyotjeannin, Morelle, Parisse (éd.) 1993 : Les cartulaires. Actes de la Table ronde organisée par l'École Nationale des Chartes et le G.D.R. 121 du C.N.R.S. (Paris, 5-7 décembre 1991), réunis par 
O. Guyotjeannin, L. Morelle et M. Parisse, Paris, 1993 (Mémoires et documents de l'École des Chartes, 39).

Hoffmann (éd.) 1980 : Chronica monasterii Casinensis, éd. H. Hoffmann, MGH, SS 34, Hanovre, 1980.

Inventaire des cartulaires 1895 : Inventaire des cartulaires conservés dans les dépôts des archives de l'État, Bruxelles, 1895.

Inventaire des cartulaires 1897 : Inventaire des cartulaires conservés en Belgique ailleurs que dans les dépôts des Archives de l'État, Bruxelles, 1897.

Inventaire des cartulaires 1899 : Inventaire des cartulaires belges conservés à l'étranger, Bruxelles, 1899.

Kehr 1977 : P. F. Kehr, Papsturkunden in Italien. Reiseberichte zur Italia Pontificia, Cité du Vatican, 1977, 6 vol.

Le Braz 1963-66 : J. Le Braz, Répertoire des cartulaires de l'ancienne France, dans Bulletin de l'IRHT, 12, 1963, p. 113-125 (Laon) ; 13, 1964-1965, p. 101-110 (Soissons) ; 14, 1966, p. 97-108 (Châlons).

Maggi Bei (éd.) 1984 : Il «Liber Floriger» di Gregorio di Catino. Parte I : Testo, éd. M. T. Maggi Bei, Rome, 1984 (Miscellanea della Società romana di Storia patria, 26).

Martin (éd.) 1987 : J.-M. Martin, Le cartulaire de S. Matteo di Sculgola en Capitanate (Registro d'istrumenti di S. Maria del Gualdo) (1177-1239), Bari, 1987, 2 vol. (Codice diplomatico Pugliese, $\mathrm{XXX} / 1-2)$.

Martin 2009 : J.-M. Martin, Occasions et modalités du remploi dans les cartulaires-chroniques de l'Italie méridionale, dans P. Toubert, P. Moret (éd.), Remploi, citation, plagiat. Conduites et pratiques médiévales (X'-XII siècle), Madrid, 2009 (Collection de la Casa de Velázquez, 112), p. 141-160.

Martin, Orofino (éd.) 2000 : Chronicon Sanctae Sophiae (cod. Vat. Lat. 4939), ed. J.-M. Martin, con uno studio di G. Orofino, Rome, 2000, 2 vol. (Fonti per la Storia dell'Italia Medievale. Rerum Italicarum Scriptores, $3 *-3 * *)$.

Mazzatinti 1890-2012 : G. Mazzatinti, Inventari dei manoscritti nelle biblioteche d'Italia, vol. 1, Forlì, 1890 - vol. 115-1, Florence, 2012.

Mazzatinti 1897-1915 : G. Mazzatinti, Gli archivi della storia d'Italia, Rocca San Casciano, 1897-1915, 9 vol.

Muratori (éd.) 1726 : L. A. Muratori, Rerum Italicarum Scriptores, II/2, Milan, 1726.

Obermair-Bitschnau 2000: H. Obermair, M. Bitschnau, Le notitiae traditionum del monastero dei canonici agostiniani di S. Michele all'Adige. Studio preliminare all'edizione della Sezione II del Tiroler Urkundenbuch, dans Studi di storia medioevale e di diplomatica, 18, 2000, p. 97-171 - Distribuito in formato digitale da Reti Medievali, URL http://rm.univr.it/biblioteca/scaffale/o.htm\#HannesObermair.

Oesterley 1886 : H. Oesterley, Wegweiser durch die Literatur der Urkundensammlungen, Berlin, 1886, 2 vol.

Olivieri 2003 : A. Olivieri, I registri vescovili nel Piemonte medievale (secoli XIII-XV). Tipologia e confronto, dans A. Bartoli Langeli, A. Rigon (éd.) I registri vescovili dell'Italia settentrionale (secoli XIII-XV). Atti del Convegno di studi (Monselice, 24-25 novembre 2000), Rome, 2003, p. 1-42.

Pio (éd.) 2001 : B. Pio, Alexandri monachi Chronicorum liber Sancti Bartholomei de Carpineto, Rome, 2001 (Fonti per la Storia dell'Italia medievale. Rerum Italicarum Scriptores, 5).

Pohl 2001 : W. Pohl, Werkstätte der Erinnerung. Montecassino und die Gestaltung der langobardischen Vergangenheit, Vienne-Munich, 2001.

Puncuh 1999 : D. Puncuh, Cartulari monastici e conventuali : confronti e osservazioni per un censimento, dans G. Avarucci, R. M. Borraccini Verducci, G. Borri (éd.), Libro, scrittura, documento della civiltà monastica e conventuale nel Basso Medioevo (secoli XIII-XV), Spolète, 1999 (Studi e ricerche, 1), p. 341-380.

Rabotti et al. (éd.) 1985 : Breviarium Ecclesiae Ravennatis (Codice Bavaro) secoli VII-X, a cura di G. Rabotti, appendici documentarie a cura di C. Curradi, G. Rabotti, A. Vasina, Rome, 1985 (Fonti per la storia d'Italia, 110).

Redlich 1884 : O. Redlich, Über bairische Traditionsbücher und Traditionen, dans Mitteilungen des Instituts für österreichische Geschichtsforschung, 5, 1884, p. 1-82.

Rovere 1984 : A. Rovere, Libri « iurium-privilegiorum, contractuum-instrumentorum » e livellari della Chiesa genovese (secc. XII-XV). Ricerche della documentazione ecclesiastica, dans Atti della Società ligure di storia patria, n. s. 24 (98)-1, 1984, p. 105-170. 
Rovere 1989 : A. Rovere, I « libri iurium » dell'Italia comunale, dans Civiltà comunale : libro, scrittura, documento. Atti del Convegno, Genova, 8-11 novembre 1988, Gênes, 1989 (Atti della Società ligure di storia patria, n. s. XXIX/2), p. 157-199.

Schiaparelli 1900 : L. Schiaparelli, Il rotolo dell'Archivio capitolare di Novara, dans Archivio storico Lombardo, 13, 1900, p. 5-48.

Soddu, Strinna (éd.) 2013 : Il condaghe di San Pietro di Silki, éd. A. Soddu et G. Strinna, Nuoro, 2013.

Späth 2007 : M. Späth, Verflechtung von Erinnerung. Bildproduktion und Geschichtsschreibung im Kloster San Clemente a Casauria während des 12. Jahrhunderts, Berlin, 2007 (Orbis mediaevalis. Vorstellungswelten des Mittelalters, Bd. 8).

Stein 1907 : H. Stein, Bibliographie générale des cartulaires français ou relatifs à l'histoire de France, Paris, 1907.

Tomei 2012 : P. Tomei, Un nuovo 'polittico' lucchese del IX secolo : il Breve de multis pensionibus, dans Studi medievali, s. III, 52-2, 2012, p. 567-602.

Vérité et al. 2003 : Répertoire des cartulaires français : provinces ecclésiastiques d'Aix, Arles, Embrun, Vienne, diocèse de Tarentaise, par I. Vérité, A.-M. Legras, C. Bourlet, A. Dufour, avec la collaboration d'O. Guyotjeannin, A.-M. Hayez, J.-L. Lemaître, M. de Valence, Paris, 2003 (Documents, études et répertoires publiés par l'IRHT, 72).

Zielinski 1972 : H. Zielinski, Studien zu den spoletinischen "Privaturkunden » des 8. Jahrhunderts und ihrer Überlieferung im Regestum Farfense, Tübingen, 1972 (Bibliothek des Deutschen Historischen Instituts in Rom, 39).

Zucchetti (éd.) 1913-1932 : Liber Largitorius vel notarius monasterii Pharphensis, éd. G. Zucchetti, Rome, 1913-1932, 2 vol. (Regesta Chartarum Italiae, 9/1-9/2).

\section{Notes}

1 Cárcel Ortí (éd.) 1997, p. 35-36 nº 74.

2 späth 2007.

3 Geary 1996, p. 140-149.

4 Martin, Orofino (éd.) 2000.

5 On peut rapprocher les cartulaires des œuvres historiques étudiées par Pohl 2001.

6 Stein, 1907.

7 Vérité et al. 2003, p. VIII.

8 Le Braz 1963-1966; ces publications sont signalées dans Vérité et al. 2003, p. IX.

9 Vérité et al. 2003. Voir également le site : www.cn-telma.fr/cartulR/index/

10 Inventaire des cartulaires 1895 . Inventaire des cartulaires 1897 . Inventaire des cartulaires 1899.

11 Davis 1958.

12 Voir, pour la Bavière, le travail ancien de Redlich 1884.

13 Oesterley 1886.

14 Ibid., II, p. 186-282 : IV. Abteilung. Italien.

15 Stein 1907, p. XI.

16 Guyotjeannin, Morelle, Parisse (éd.) 1993.

17 Voir Martin 2009. Au colloque de Paris, une seule intervention concernait un cartulaire italien : Feller 1993.

18 Giorgi, Balzani (éd.) 1879-1914. Sur la datation des œuvres de Grégoire, voir Zielinski 1972, p. 26-28.

19 Zucchetti (éd.) 1913-1932.

20 Balzani (éd.) 1903.

21 Maggi Bei (éd.) 1984.

22 Hoffmann (éd.) 1980.

23 Un fac-similé a été publié au Mont-Cassin en 2000, l'édition est sous presse.

24 Martin, Orofino (éd.) 2000.

25 Federici (éd.) 1925-1940. 
26 L'édition, préparée par le regretté Alessandro Pratesi, doit paraître prochainement. Le Chronicon Casauriense n'avait encore fait l'objet que d'une édition partielle dans Muratori (éd.) 1726, c. 767-1018.

27 Fuselli (éd.) 1996. Pio (éd.), 2001.

28 Rovere 1989.

29 De Bianchi 2013.

30 La grande majorité des cartulaires italiens se trouve en Italie. Mais on sait que le Chronicon Casauriense est conservé à Paris ; certains de ceux qui concernent le Trentin-Haut Adige (région qui connaît le modèle des «Traditionsbücher » de l'Allemagne méridionale) sont à Munich, et le fonds Ashburnham de la British Library possède deux cartulaires (un de Toscane, un de Vérone).

31 Voir notamment : Rovere 1984 ; Obermair-Bitschnau 2000 ; Olivieri 2003 ; Cameli 2002 ; Cameli 2003 ; Cameli 2005 ; Allegria 2006 ; Cameli 2009 (le second chapitre de cet ouvrage est consacré à la documentation fournie par les registres, et en particulier au Liber quartus, cartulaire épiscopal commencé en 1322) ; Soddu, Strinna (éd.) 2013.

32 Sur l'importance et la signification de la décoration, on se réfère notamment (mais pas exclusivement) au récent travail de späth 2007.

33 Kehr 1977. Mazzatinti 1897-1915. Mazzatinti 1890-2012 (la collection, parvenue au vol. 115-1, se poursuit).

34 Il ne semble pas que cette pratique ait gagné l'Italie méridionale. Notons toutefois que, dans le cartulaire de S. Maria del Gualdo, établi vers 1225 , le compilateur a pris soin de reproduire avec précision les signa des notaires qui avaient écrit les actes : Martin (éd.) 1987.

35 Rovere 1984. Puncuh 1999.

36 Rabotti et al. (éd.) 1985.

37 Tomei 2012 ; il s'agit en fait d'un cartulaire, non d'un polyptyque.

38 Carbonetti Vendittelli 2011.

39 Schiaparelli 1900.

40 Guillou (éd.) 1972.

\section{Pour citer cet article}

Référence électronique

Cristina Carbonetti, Arianna Cervi, Marta De Bianchi et Jean-Marie Martin, « Les cartulaires ecclésiastiques de l'Italie médiévale », Mélanges de l'École française de Rome - Moyen Âge [En ligne], 127-2 | 2015, mis en ligne le 16 octobre 2015, consulté le 29 octobre 2015. URL : http:// mefrm.revues.org/2655

\section{À propos des auteurs}

\section{Cristina Carbonetti}

Università degli studi di Roma Tor Vergata - cristina.carbonetti@uniroma2.it

Arianna Cervi

Università degli studi di Milano - gemini.86@ hotmail.it

Marta De Bianchi

Università degli studi di Roma Tor Vergata - martadebianchi@gmail.com

Jean-Marie Martin

CNRS, Paris - martin-hisard@wanadoo.fr

\section{Droits d'auteur}

(C) École française de Rome 


\section{Résumés}

Les cartulaires, dont le contenu est depuis longtemps utilisé comme source de l'histoire du Moyen Âge, sont à présent considérés aussi comme des œuvres originales. Ils ont été bien étudiés en France et dans quelques autres pays, mais il n'existe pas de répertoire des cartulaires italiens. L'Italie a produit d'une part des cartulaires (parfois accompagnés d'une chronique) compilés, surtout au XII ${ }^{\mathrm{e}}$ siècle, par les églises elles-mêmes, d'autre part, plus tard, des recueils documentaires faits par des notaires sur le modèle des libri iurium communaux. Nous préparons un catalogue général des cartulaires ecclésiastiques italiens, chacun faisant l'objet d'une fiche typologique ; nous avons déjà identifié un troisième type de cartulaire (le plus ancien), recensant non les possessions, mais les concessions.

The cartularies have been used for a long time only as a historical source, but now they are considered as original works and their production and distribution are studied as a particular documentary phenomenon and as a manifestation of a peculiar practice of writing. They have been studied in France and in other countries, but there is still no repertory of Italian ecclesiastical cartularies. During the Middle Ages Italian religious institutions produced (especially in $11^{\text {th }}$ and $12^{\text {th }}$ centuries) cartularies compiled by their scribes (sometimes accompanied by chronicles) and, then (from the $13^{\text {th }}$ century), documentary collections drawn up by notaries on the model of municipal libri iurium. This research aims 1) at drawing up a catalog of Italian ecclesiastical cartularies, where each cartulary will be described in detail on the basis of a card model, and 2) at studying the phenomenon of production of ecclesiastical cartularies in its various typological and regional occurrences.

\section{Entrées d'index}

Mots-clés : Cartulaires ecclésiastiques, Chroniques, Libri iurium Keywords : Ecclesiastical Cartularies, Chronicles, Libri iurium 